\title{
FEKETE-SZEGÖ INEQUALITIES FOR CERTAIN SUBCLASS OF ANALYTIC FUNCTIONS DEFINED BY USING SALAGEAN OPERATOR
}

\author{
R. M. EL-ASHWAH AND A. H. HASSAN \\ Received 14 January, 2015
}

\begin{abstract}
In this paper, we obtain some coefficients bounds (namely, Fekete-Szegö inequalities) for functions belonging to certain subclass of analytic functions defined by using Salagean operator. Moreover, connections of the results presented here with those obtained in earlier works are pointed out.
\end{abstract}

2010 Mathematics Subject Classification: $30 \mathrm{C} 45$

Keywords: analytic, starlike functions, convex functions, subordination, Fekete-Szegö problem

\section{INTRODUCTION}

Let $\mathcal{A}$ denotes the class of functions $f(z)$ of the form:

$$
f(z)=z+\sum_{k=2}^{\infty} a_{k} z^{k}(z \in U)
$$

which are analytic in the open unit disc $U=\{z: z \in \mathbb{C}$ and $|z|<1\}$. Further let $S$ denote the family of functions of the form (1.1) which are univalent in $U$. A classical theorem of Fekete-Szegö [6] states that, for $f(z) \in S$ given by (1.1) that

$$
\left|a_{3}-\mu a_{2}^{2}\right| \leq\left\{\begin{array}{cc}
3-4 \mu, & \text { if } \mu \leq 0, \\
1+2 \exp \left(\frac{-2 \mu}{1-\mu}\right), & \text { if } 0 \leq \mu \leq 1, \\
4 \mu-3, & \text { if } \mu \geq 1 .
\end{array}\right.
$$

The result is sharp.

Given two functions $f(z)$ and $g(z)$, which are analytic in $U$ with $f(0)=g(0)$, the function $f(z)$ is said to be subordinate to $g(z)$ in $U$ if there exists a function $w(z)$, analytic in $U$, such that $w(0)=0$ and $|w(z)|<1(z \in U)$ and $f(z)=$ $g(w(z))(z \in U)$. We denote this subordination by $f(z) \prec g(z)$ in $U$ (see [11]).

Let $\varphi(z)$ be an analytic function with positive real part on $U$, which satisfies $\varphi(0)=$ 1 and $\varphi^{\prime}(0)>0$, and which maps the unit disc $U$ onto a region starlike with respect 
to 1 and symmetric with respect to the real axis. Let $S^{*}(\varphi)$ be the class of functions $f(z) \in S$ for which

$$
\frac{z f^{\prime}(z)}{f(z)} \prec \varphi(z)(z \in U)
$$

and $C(\varphi)$ be the class of functions $f(z) \in S$ for which

$$
1+\frac{z f^{\prime \prime}(z)}{f^{\prime}(z)} \prec \varphi(z)(z \in U) .
$$

The classes of $S^{*}(\varphi)$ and $C(\varphi)$ were introduced and studied by Ma and Minda [10]. The familier class $S^{*}(\alpha)$ of starlike functions of order $\alpha$ and the class $C(\alpha)$ of convex functions of order $\alpha(0 \leq \alpha<1)$ are the special cases of $S^{*}(\varphi)$ and $C(\varphi)$, respectively, when $\varphi(z)=\frac{1+(1-2 \alpha) z}{1-z}(0 \leq \alpha<1)$.

Ma and Minda [10] have obtained the Fekete-Szegö problem for the functions in the class $C(\varphi)$.

For a function $f(z) \in \mathcal{S}$, Salagean [18] introduced the following differential operator as following:

$$
D^{0} f(z)=f(z), D^{1} f(z)=z f^{\prime}(z), \ldots, D^{n} f(z)=D\left(D^{n-1} f(z)\right)(n \in \mathbb{N}=\{1,2, \ldots\}) .
$$

We note that

$$
D^{n} f(z)=z+\sum_{k=2}^{\infty} k^{n} a_{k} z^{k}\left(n \in \mathbb{N}_{0}=\mathbb{N} \cup\{0\}\right) .
$$

Using Salagean operator we introduce a new class of analytic functions as following:

Definition 1. For $b \in \mathbb{C}^{*}=\mathbb{C} \backslash\{0\}$, the class $M_{\alpha, n}^{b}(\varphi)$ consists of all functions $f(z) \in \mathcal{A}$ satisfying the following subordination:

$$
1+\frac{1}{b}\left(\frac{z\left(D^{n} f(z)\right)^{\prime}+\alpha z^{2}\left(D^{n} f(z)\right)^{\prime \prime}}{(1-\alpha) D^{n} f(z)+\alpha z\left(D^{n} f(z)\right)^{\prime}}-1\right) \prec \varphi(z),\left(\alpha \geq 0 ; n \in \mathbb{N}_{0} ; z \in U\right) .
$$

Specializing the parameters $\alpha, b, n$, and $\varphi(z)$, we obtain the following subclasses studied by various authors:

(i) $M_{\alpha, n}^{1}(\varphi)=M_{\alpha, n}(\varphi)$ (see Orhan and Gunes [16]);

(ii) $M_{0, n}^{b}(\varphi)=H_{n, b}(\varphi)$ (see Aouf and Silverman [3]);

(iii) $M_{\alpha, 0}^{1}(\varphi)=M_{\alpha}(\varphi)$ (see Shanmugam and Sivasubramanian [19]);

(iv) $M_{0, n}^{b}\left(\frac{1+A z}{1+B z}\right)=H_{n}^{b}(A, B)(-1 \leq B<A \leq 1)$ (see Attiya [4]);

(v) $M_{0,0}^{b}(\varphi)=S_{b}^{*}(\varphi)$ and $G_{0,1}^{b}(\varphi)=C_{b}(\varphi)$ (see Ravichandran et al. [17]);

(vi) $M_{0, n}^{b}\left(\frac{1+z}{1-z}\right)=S^{n}(b)$ (see Aouf et al. [1]); 
(vii) $M_{0,0}^{b}\left(\frac{1+z}{1-z}\right)=S(b)$ (see Nasr and Aouf [14,15] see also Aouf et al. [2]);

(viii) $M_{0,1}^{b}\left(\frac{1+z}{1-z}\right)=C(b)$ (see Nasr and Aouf [12,13] see also Aouf et al. [2]);

(ix) $M_{0,0}^{(1-\rho) \cos \eta e^{-i \eta}}\left(\frac{1+z}{1-z}\right)=S^{\eta}(\rho)\left(|\eta|<\frac{\pi}{2}, 0 \leq \rho<1\right)$ (see Libera [9] see also Keogh and Merkes [8]);

(x) $M_{0,1}^{(1-\rho) \cos \eta e^{-i \eta}}\left(\frac{1+z}{1-z}\right)=C^{\eta}(\rho)\left(|\eta|<\frac{\pi}{2}, 0 \leq \rho<1\right)$ (see Chichra [5]).

Also we note that for additional choices of parameters we have the following new subclasses of $\mathcal{A}$ :

(i) $M_{1, n}^{b}(\varphi)=N_{n}^{b}(\varphi)=\left\{f(z) \in \mathcal{A}: 1+\frac{1}{b} \frac{z\left(D^{n} f(z)\right)^{\prime \prime}}{\left(D^{n} f(z)\right)^{\prime}} \prec \varphi(z)\left(n \in \mathbb{N}_{0} ; b \in \mathbb{C}^{*} ; z \in U\right)\right\}$;

(ii) $M_{\alpha, n}^{(1-\rho) \cos \eta e^{-i \eta}}(\varphi)=M_{\alpha, n}^{\rho, \eta}(\varphi)$

$$
=\left\{\begin{array}{r}
f(z) \in \mathcal{A}: \frac{e^{i \eta} \frac{z\left(D^{n} f(z)\right)^{\prime}+\alpha z^{2}\left(D^{n} f(z)\right)^{\prime \prime}}{(1-\alpha) D^{n} f(z)+\alpha z\left(D^{n} f(z)\right)^{\prime}}-\rho \cos \eta-i \sin \eta}{(1-\rho) \cos \eta} \prec \varphi(z) \\
\left.\left(|\eta|<\frac{\pi}{2} ; \alpha \geq 0 ; 0 \leq \rho<1 ; n \in \mathbb{N}_{0} ; z \in U\right)\right\} .
\end{array}\right.
$$

In this paper, we obtain the Fekete-Szegö inequalities for functions in the class $M_{\alpha, n}^{b}(\varphi)$.

\section{FEKETE-SZEGÖ PROBLEM}

Unless otherwise mentioned, we assume in the reminder of this paper that $\alpha \geq 0$, $b \in \mathbb{C}^{*}, n \in \mathbb{N}_{0}$ and $z \in U$.

To prove our results, we shall need the following lemmas (see [10]):

Lemma 1. If $p(z)=1+c_{1} z+c_{2} z^{2}+\ldots$ is a function with positive real part in $U$ and $\mu$ is a complex number, then

$$
\left|c_{2}-\mu c_{1}^{2}\right| \leq 2 \max \{1 ;|2 \mu-1|\} .
$$

The result is sharp for the functions given by

$$
p(z)=\frac{1+z^{2}}{1-z^{2}} \text { and } p(z)=\frac{1+z}{1-z}(z \in U) .
$$

Lemma 2. If $p_{1}(z)=1+c_{1} z+c_{2} z^{2}+\ldots$ is a function with positive real part in $U$, then

$$
\left|c_{2}-v c_{1}^{2}\right| \leq\left\{\begin{array}{lc}
-4 v+2, & \text { if } v \leq 0 \\
2, & \text { if } 0 \leq v \leq 1, \\
4 v-2, & \text { if } v \geq 1
\end{array}\right.
$$


When $v<0$ or $v>1$, the equality holds if and only if $p_{1}(z)=\frac{1+z}{1-z}$ or one of its rotations. If $0<v<1$, then the equality holds if and only if $p_{1}(z)=\frac{1+z^{2}}{1-z^{2}}$ or one of its rotations. If $v=0$, the equality holds if and only if

$$
p_{1}(z)=\left(\frac{1}{2}+\frac{1}{2} \gamma\right) \frac{1+z}{1-z}+\left(\frac{1}{2}-\frac{1}{2} \gamma\right) \frac{1-z}{1+z}(0 \leq \gamma \leq 1),
$$

or one of its rotations. If $v=1$, the equality holds if and only if

$$
\frac{1}{p_{1}(z)}=\left(\frac{1}{2}+\frac{1}{2} \gamma\right) \frac{1+z}{1-z}+\left(\frac{1}{2}-\frac{1}{2} \gamma\right) \frac{1-z}{1+z}(0 \leq \gamma \leq 1) .
$$

Also the above upper bound is sharp and it can be improved as follows when $0<v<$ 1 :

and

$$
\left|c_{2}-v c_{1}^{2}\right|+v\left|c_{1}\right|^{2} \leq 2\left(0<v<\frac{1}{2}\right)
$$

$$
\left|c_{2}-v c_{1}^{2}\right|+(1-v)\left|c_{1}\right|^{2} \leq 2\left(\frac{1}{2}<v<1\right)
$$

Using Lemma 1, we have the following theorem:

Theorem 1. Let $\varphi(z)=1+B_{1} z+B_{2} z^{2}+B_{3} z^{3}+\ldots$, where $\varphi(z) \in \mathcal{A}$ and $\varphi^{\prime}(0)>0$. If $f(z)$ given by (1.1) belongs to the class $M_{\alpha, n}^{b}(\varphi)$ and if $\mu$ is a complex number, then

$$
\left|a_{3}-\mu a_{2}^{2}\right| \leq \frac{|b| B_{1}}{2.3^{n}(1+2 \alpha)} \max \left\{1,\left|\frac{B_{2}}{B_{1}}+\left(1-\frac{3^{n}(1+2 \alpha)}{2^{2 n-1}(1+\alpha)^{2}} \mu\right) b B_{1}\right|\right\} .
$$

The result is sharp.

Proof. If $f(z) \in M_{\alpha, n}^{b}(\varphi)$, then there exists a Schwarz function $w(z)$ which is analytic in $U$ with $w(0)=0$ and $|w(z)|<1$ in $U$ and such that

$$
1+\frac{1}{b}\left(\frac{z\left(D^{n} f(z)\right)^{\prime}+\alpha z^{2}\left(D^{n} f(z)\right)^{\prime \prime}}{(1-\alpha) D^{n} f(z)+\alpha z\left(D^{n} f(z)\right)^{\prime}}-1\right)=\varphi(w(z)) .
$$

Define the function $p_{1}(z)$ by

$$
p_{1}(z)=\frac{1+w(z)}{1-w(z)}=1+c_{1} z+c_{2} z^{2}+\ldots .
$$

Since $w(z)$ is a Schwarz function, we see that $\Re\left\{p_{1}(z)\right\}>0$ and $p_{1}(0)=1$. Define the function $p(z)$ by:

$$
p(z)=1+\frac{1}{b}\left(\frac{z\left(D^{n} f(z)\right)^{\prime}+\alpha z^{2}\left(D^{n} f(z)\right)^{\prime \prime}}{(1-\alpha) D^{n} f(z)+\alpha z\left(D^{n} f(z)\right)^{\prime}}-1\right)=1+b_{1} z+b_{2} z^{2}+\ldots .
$$


In view of the equations (2.4), (2.5) and (2.6), we have

$$
\begin{aligned}
p(z) & =\varphi\left(\frac{p_{1}(z)-1}{p_{1}(z)+1}\right)=\varphi\left(\frac{c_{1} z+c_{2} z^{2}+\ldots}{2+c_{1} z+c_{2} z^{2}+\ldots}\right) \\
& =\varphi\left(\frac{1}{2} c_{1} z+\frac{1}{2}\left(c_{2}-\frac{c_{1}^{2}}{2}\right) z^{2}+\ldots\right) \\
& =1+\frac{1}{2} B_{1} c_{1} z+\left[\frac{1}{2} B_{1}\left(c_{2}-\frac{c_{1}^{2}}{2}\right)+\frac{1}{4} B_{2} c_{1}^{2}\right] z^{2}+\ldots
\end{aligned}
$$

Thus

$$
b_{1}=\frac{1}{2} B_{1} c_{1} \quad \text { and } \quad b_{2}=\frac{1}{2} B_{1}\left(c_{2}-\frac{c_{1}^{2}}{2}\right)+\frac{1}{4} B_{2} c_{1}^{2} .
$$

Since

$$
\begin{aligned}
1+\frac{1}{b}\left(\frac{z\left(D^{n} f(z)\right)^{\prime}+\alpha z^{2}\left(D^{n} f(z)\right)^{\prime \prime}}{(1-\alpha) D^{n} f(z)+\alpha z\left(D^{n} f(z)\right)^{\prime}}-1\right)=1+\left\{\frac{1}{b} 2^{n}(1+\alpha) a_{2}\right\} z & \\
+ & \left\{\frac{2.3^{n}(1+2 \alpha) a_{3}-2^{2 n}(1+\alpha)^{2} a_{2}^{2}}{b}\right\} z^{2}+\ldots .
\end{aligned}
$$

Then from 2.6 and 2.8, we obtain

$$
a_{2}=\frac{b B_{1} c_{1}}{2^{n+1}(1+\alpha)}
$$

and

$$
a_{3}=\frac{b B_{1} c_{2}}{4.3^{n}(1+2 \alpha)}+\frac{c_{1}^{2}}{8.3^{n}(1+2 \alpha)}\left[b^{2} B_{1}^{2}-b\left(B_{1}-B_{2}\right)\right] .
$$

Therefore, we have

$$
a_{3}-\mu a_{2}^{2}=\frac{b B_{1}}{4.3^{n}(1+2 \alpha)}\left[c_{2}-v c_{1}^{2}\right]
$$

where

$$
v=\frac{1}{2}\left[1-\frac{B_{2}}{B_{1}}+\left(\frac{3^{n}(1+2 \alpha)}{2^{2 n-1}(1+\alpha)^{2}} \mu-1\right) b B_{1}\right] .
$$

Our result now follows by an application of Lemma 1 . The result is sharp for the functions

$$
1+\frac{1}{b}\left(\frac{z\left(D^{n} f(z)\right)^{\prime}+\alpha z^{2}\left(D^{n} f(z)\right)^{\prime \prime}}{(1-\alpha) D^{n} f(z)+\alpha z\left(D^{n} f(z)\right)^{\prime}}-1\right)=\varphi\left(z^{2}\right)
$$

and

$$
1+\frac{1}{b}\left(\frac{z\left(D^{n} f(z)\right)^{\prime}+\alpha z^{2}\left(D^{n} f(z)\right)^{\prime \prime}}{(1-\alpha) D^{n} f(z)+\alpha z\left(D^{n} f(z)\right)^{\prime}}-1\right)=\varphi(z) .
$$

This completes the proof of Theorem 1 . 
Remark 1. (i) Taking $\alpha=n=0$ in Theorem 1, we improve the result obtained by Ravichandran et al. [17, Theorem 4.1];

(ii) Taking $\alpha=0, b=(1-\rho) \cos \eta e^{-i \eta}\left(|\eta|<\frac{\pi}{2}, 0 \leq \rho<1\right)$ and $\varphi(z)=\frac{1+z}{1-z}$ (equivalently $B_{1}=B_{2}=2$ ) in Theorem 1, we obtain the result obtained by Goyal and Kumar [7, Corollary 2.10];

(iii) Taking $\alpha=n=0, b=(1-\rho) \cos \eta e^{-i \eta}\left(|\eta|<\frac{\pi}{2}, 0 \leq \rho<1\right)$ and $\varphi(z)=\frac{1+z}{1-z}$ in Theorem 1, we obtain the result obtained by Keogh and Merkes [8, Theorem 1];

(iv) Taking $\alpha=0$ in Theorem 1, we obtain the result obtained by Aouf and Silverman [3, Theorem 1].

Using Lemma 2, we obtain the following theorem:

Theorem 2. Let $\varphi(z)=1+B_{1} z+B_{2} z^{2}+B_{3} z^{3}+\ldots,\left(b>0 ; B_{i}>0 ; i \in \mathbb{N}\right)$.Also let

$$
\sigma_{1}=\frac{2^{2 n-1}(1+\alpha)^{2}\left(b B_{1}^{2}+B_{2}-B_{1}\right)}{3^{n}(1+2 \alpha) b B_{1}^{2}}
$$

and

$$
\sigma_{2}=\frac{2^{2 n-1}(1+\alpha)^{2}\left(b B_{1}^{2}+B_{2}+B_{1}\right)}{3^{n}(1+2 \alpha) b B_{1}^{2}} .
$$

If $f(z)$ is given by (1.1) belongs to the class $M_{\alpha, n}^{b}(\varphi)$, then we have the following sharp results:

(i) If $\mu \leq \sigma_{1}$, then

$$
\left|a_{3}-\mu a_{2}^{2}\right| \leq \frac{b}{2.3^{n}(1+2 \alpha)}\left[B_{2}-\left(\frac{3^{n}(1+2 \alpha)}{2^{2 n-1}(1+\alpha)^{2}} \mu-1\right) b B_{1}^{2}\right]
$$

(ii) If $\sigma_{1} \leq \mu \leq \sigma_{2}$, then

$$
\left|a_{3}-\mu a_{2}^{2}\right| \leq \frac{b}{2.3^{n}(1+2 \alpha)}
$$

(iii) If $\mu \geq \sigma_{2}$, then

$$
\left|a_{3}-\mu a_{2}^{2}\right| \leq \frac{b}{2.3^{n}(1+2 \alpha)}\left[-B_{2}+\left(\frac{3^{n}(1+2 \alpha)}{2^{2 n-1}(1+\alpha)^{2}} \mu-1\right) b B_{1}^{2}\right] .
$$

Proof. For $f(z) \in M_{\alpha, n}^{b}(\varphi), p(z)$ given by (2.6) and $p_{1}(z)$ given by (2.5), then $a_{2}$ and $a_{3}$ are given as same as in Theorem 1. Also

$$
a_{3}-\mu a_{2}^{2}=\frac{b B_{1}}{4.3^{n}(1+2 \alpha)}\left[c_{2}-v c_{1}^{2}\right],
$$

where $v$ is given by (2.12).

First, if $\mu \leq \sigma_{1}$, then we have $v \leq 0$, then by applying Lemma 2 to equality (2.18), we have

$$
\left|a_{3}-\mu a_{2}^{2}\right| \leq \frac{b}{2.3^{n}(1+2 \alpha)}\left[B_{2}-\left(\frac{3^{n}(1+2 \alpha)}{2^{2 n-1}(1+\alpha)^{2}} \mu-1\right) b B_{1}^{2}\right],
$$


which is evidently inequality (2.15) of Theorem 2 .

If $\mu=\sigma_{1}$, then we have $v=0$, therefore equality holds if and only if

$$
p_{1}(z)=\left(\frac{1+\gamma}{2}\right) \frac{1+z}{1-z}+\left(\frac{1-\gamma}{2}\right) \frac{1-z}{1+z}(0 \leq \gamma \leq 1 ; z \in U) .
$$

Next, if $\sigma_{1} \leq \mu \leq \sigma_{2}$, we note that

$$
\max \left\{\frac{1}{2}\left[1-\frac{B_{2}}{B_{1}}+\left(\frac{3^{n}(1+2 \alpha)}{2^{2 n-1}(1+\alpha)^{2}} \mu-1\right) b B_{1}\right]\right\} \leq 1,
$$

then applying Lemma 2 to equality (2.18), we have

$$
\left|a_{3}-\mu a_{2}^{2}\right| \leq \frac{b}{2.3^{n}(1+2 \alpha)}
$$

which is evidently inequality (2.16) of Theorem 2 .

If $\sigma_{1}<\mu<\sigma_{2}$, then we have

$$
p_{1}(z)=\frac{1+z^{2}}{1-z^{2}}
$$

Finally, If $\mu \geq \sigma_{2}$, then we have $v \geq 1$, therefore by applying Lemma 2 to (2.18), we have

$$
\left|a_{3}-\mu a_{2}^{2}\right| \leq \frac{b}{2.3^{n}(1+2 \alpha)}\left[\left(\frac{3^{n}(1+2 \alpha)}{2^{2 n-1}(1+\alpha)^{2}} \mu-1\right) b B_{1}^{2}-B_{2}\right],
$$

which is evidently inequality (2.17) of the theorem. If $\mu=\sigma_{2}$, then we have $v=1$, therefore equality holds if and only if

$$
\frac{1}{p_{1}(z)}=\left(\frac{1+\gamma}{2}\right) \frac{1+z}{1-z}+\left(\frac{1-\gamma}{2}\right) \frac{1-z}{1+z}(0 \leq \gamma \leq 1 ; z \in U) \text {. }
$$

To show that the bounds are sharp, we define the functions $K_{\varphi}^{s}(s \geq 2)$ by

$$
1+\frac{1}{b}\left(\frac{z\left(D^{n} K_{\varphi}^{s}(z)\right)^{\prime}+\alpha z^{2}\left(D^{n} K_{\varphi}^{s}(z)\right)^{\prime \prime}}{(1-\alpha) D^{n} K_{\varphi}^{s}(z)+\alpha z\left(D^{n} K_{\varphi}^{s}(z)\right)^{\prime}}-1\right)=\varphi\left(z^{s-1}\right), K_{\varphi}^{s}(0)=0=K_{\varphi}^{\prime s}(0)-1,
$$

and the functions $F_{t}$ and $G_{t}(0 \leq t \leq 1)$ by

$1+\frac{1}{b}\left(\frac{z\left(D^{n} F_{t}(z)\right)^{\prime}+\alpha z^{2}\left(D^{n} F_{t}(z)\right)^{\prime \prime}}{(1-\alpha) D^{n} F_{t}(z)+\alpha z\left(D^{n} F_{t}(z)\right)^{\prime}}-1\right)=\varphi\left(\frac{z(z+t)}{1+t z}\right), F_{t}(0)=0=F_{t}^{\prime}(0)-1$,

and

$$
1+\frac{1}{b}\left(\frac{z\left(D^{n} G_{t}(z)\right)^{\prime}+\alpha z^{2}\left(D^{n} G_{t}(z)\right)^{\prime \prime}}{(1-\alpha) D^{n} G_{t}(z)+\alpha z\left(D^{n} G_{t}(z)\right)^{\prime}}-1\right)=\varphi\left(-\frac{z(z+t)}{1+t z}\right), G_{t}(0)=0=G_{t}^{\prime}(0)-1 .
$$

Cleary, the functions $K_{\varphi}^{s}, F_{t}$ and $G_{t} \in M_{\alpha, n}^{b}(\varphi)$. Also we write $K_{\varphi}=K_{\varphi}^{2}$. 
If $\mu<\sigma_{1}$ or $\mu>\sigma_{2}$, then the equality holds if and only if $f$ is $K_{\varphi}$ or one of its rotations. When $\sigma_{1}<\mu<\sigma_{2}$, then the equality holds if $f$ is $K_{\varphi}^{3}$ or one of its rotations. If $\mu=\sigma_{1}$, then the equality holds if and only if $f$ is $F_{t}$ or one of its rotations. If $\mu=\sigma_{2}$, then the equality holds if and only if $f$ is $G_{t}$ or one of its rotations.

Remark 2. (i) Taking $b=1$ and $n=0$ in Theorem 2, we improve the result obtained by Shanmugam and Sivasubramanian [19, Theorem 2.1];

(ii) Taking $b=1$ in Theorem 2, we obtain the result obtained by Orhan and Gunes [16, Theorem 2.1];

(iii) Taking $\alpha=0$ in Theorem 2, we obtain the result obtained by Aouf and Silverman [3, Theorem 2].

Also, using Lemma 2 we have the following theorem:

Theorem 3. For $\varphi(z)=1+B_{1} z+B_{2} z^{2}+B_{3} z^{3}+\ldots,\left(b>0 ; B_{i}>0 ; i \in \mathbb{N}\right)$ and $f(z)$ given by (1.1) belongs to the class $M_{\alpha, n}^{b}(\varphi)$ and $\sigma_{1} \leq \mu \leq \sigma_{2}$, then in view of Lemma 2, Theorem 2 can be improved. Let

$$
\sigma_{3}=\frac{2^{2 n-1}(1+\alpha)^{2}\left(b B_{1}^{2}+B_{2}\right)}{3^{n}(1+2 \alpha) b B_{1}^{2}},
$$

(i) If $\sigma_{1} \leq \mu \leq \sigma_{3}$, then

$$
\left|a_{3}-\mu a_{2}^{2}\right|+\frac{2^{2 n-1}(1+\alpha)^{2}}{3^{n}(1+2 \alpha) b B_{1}^{2}}\left[B_{1}-B_{2}+\frac{3^{n} \mu(1+2 \alpha)-2^{2 n-1}(1+\alpha)^{2}}{2^{2 n-1}(1+\alpha)^{2}} b B_{1}^{2}\right]\left|a_{2}\right|^{2} \leq \frac{b B_{1}}{2.3^{n}(1+2 \alpha)} \text {; }
$$

(ii) If $\sigma_{3} \leq \mu \leq \sigma_{2}$, then

$$
\left|a_{3}-\mu a_{2}^{2}\right|+\frac{2^{2 n-1}(1+\alpha)^{2}}{3^{n}(1+2 \alpha) b B_{1}^{2}}\left[B_{1}+B_{2}-\frac{3^{n} \mu(1+2 \alpha)-2^{2 n-1}(1+\alpha)^{2}}{2^{2 n-1}(1+\alpha)^{2}} b B_{1}^{2}\right]\left|a_{2}\right|^{2} \leq \frac{b B_{1}}{2.3^{n}(1+2 \alpha)} .
$$

Proof. For the values of $\sigma_{1} \leq \mu \leq \sigma_{3}$, we have

$$
\begin{aligned}
\left|a_{3}-\mu a_{2}^{2}\right|+\left(\mu-\sigma_{1}\right)\left|a_{2}\right|^{2} & =\frac{b B_{1}}{4.3^{n}(1+2 \alpha)}\left|c_{2}-v c_{1}^{2}\right| \\
& +\left(\mu-\frac{2^{2 n-1}(1+\alpha)^{2}\left(b B_{1}^{2}+B_{2}-B_{1}\right)}{3^{n}(1+2 \alpha) b B_{1}^{2}}\right) \frac{b^{2} B_{1}^{2}}{2^{2 n+2}(1+\alpha)^{2}}\left|c_{1}\right|^{2} \\
& =\frac{b B_{1}}{2.3^{n}(1+2 \alpha)}\left\{\frac{1}{2}\left(\left|c_{2}-v c_{1}^{2}\right|+v\left|c_{1}\right|^{2}\right)\right\}
\end{aligned}
$$

Now apply Lemma 2 to equality (2.25), then we have

$$
\left|a_{3}-\mu a_{2}^{2}\right|+\left(\mu-\sigma_{1}\right)\left|a_{2}\right|^{2} \leq \frac{b B_{1}}{2.3^{n}(1+2 \alpha)},
$$

which is evidently inequality (2.23) of Theorem 3 . 
Next, for the values of $\sigma_{3} \leq \mu \leq \sigma_{2}$, we have

$$
\begin{aligned}
\left|a_{3}-\mu a_{2}^{2}\right|+\left(\sigma_{2}-\mu\right)\left|a_{2}\right|^{2} & =\frac{b B_{1}}{4.3^{n}(1+2 \alpha)}\left|c_{2}-v c_{1}^{2}\right| \\
& +\left(\frac{2^{2 n-1}(1+\alpha)^{2}\left(b B_{1}^{2}+B_{2}+B_{1}\right)}{3^{n}(1+2 \alpha) b B_{1}^{2}}-\mu\right) \frac{b^{2} B_{1}^{2}}{2^{2 n+2}(1+\alpha)^{2}}\left|c_{1}\right|^{2} \\
& =\frac{b B_{1}}{2 \cdot 3^{n}(1+2 \alpha)}\left\{\frac{1}{2}\left(\left|c_{2}-v c_{1}^{2}\right|+(1-v)\left|c_{1}\right|^{2}\right)\right\} .
\end{aligned}
$$

Now apply Lemma 2 to equality (2.26), then we have

$$
\left|a_{3}-\mu a_{2}^{2}\right|+\left(\sigma_{2}-\mu\right)\left|a_{2}\right|^{2} \leq \frac{b B_{1}}{2.3^{n}(1+2 \alpha)} .
$$

Which is inequality (2.24). This completes the proof of Theorem 3.

Remark 3. (i) Taking $b=1$ and $n=0$ in Theorem 3, we improve the result obtained by Shanmugam and Sivasubramanian [19, Remark 2.2];

(ii) taking $\alpha=0$ in Theorem 3, we improve the result obtained by Goyal and Kumar [7, Remark 2.8].

\section{ACKNOWLEDGEMENT}

The authors would like to thank the referees for their valuable comments that improved this paper.

\section{REFERENCES}

[1] M. K. Aouf, H. E. Darwish, and A. A. Attiya, "On a class of certain analytic functions of complex order.” Indian J. Pure Appl. Math., vol. 32, no. 10, pp. 1443-1452, 2001.

[2] M. K. Aouf, S. Owa, and M. Obradovic, "Certain classes of analytic functions of complex order and type beta." Rend Mat., vol. 11, pp. 691-714, 1991.

[3] M. K. Aouf and H. Silverman, "Fekete-Szegö inequality for n-starlike functions of complex order." Adv. Math. Sci. J., pp. 1-12, 2008.

[4] A. A. Attiya, "On a generalization class of bounded starlike functions of complex order." Appl. Math. Comput., vol. 187, pp. 62-67, 2007, doi: 10.1016/j.amc.2006.08.103.

[5] P. N. Chichra, "Regular functions $f(z)$ for which $z f^{\prime}(z)$ is $\alpha$-spirallike." Proc. Amer. Math. Soc., vol. 49, pp. 151-160, 1975, doi: 10.2307/2039807.

[6] M. Fekete and G. Szegö, "Eine bemerkung uber ungerade schlichte funktionen.” J. London Math. Soc., vol. 8, pp. 85-89, 1933, doi: 10.1112/jlms/s1-8.2.85.

[7] S. P. Goyal and S. Kumar, "Fekete-Szegö problem for a class of complex order related to Salagean operator.” Bull. Math. Anal. Appl., vol. 3, no. 4, pp. 240-246, 2011.

[8] F. R. Keogh and E. P. Merkes, "A coefficient inequality for certain classes of analytic functions." Proc. Amer. Math. Soc., vol. 20, no. 1, pp. 8-12, 1969, doi: 10.2307/2035949.

[9] R. J. Libera, “Univalent $\alpha$-spiral functions.” Canad. J. Math., vol. 19, pp. 449-456, 1967.

[10] W. Ma and D. Minda, " A unified treatment of some special classes of univalent functions. Proceedings of the conference on complex Analysis, Z. Li, F. Ren, L. Lang and S. Zhang (Eds.), Int. Press,”, pp. 157-169, 1994.

[11] S. S. Miller and P. T. Mocanu, Differential Subordinations: Theory and Applications. New York: Series on Monographs and Textbooks in Pure and Appl. Math. No. 255: Marcel Dekker, 2000. 
[12] M. A. Nasr and M. K. Aouf, "On convex functions of complex order.” Bull. Fac. Sci. Mansoura Univ., vol. 9, pp. 565-582, 1982.

[13] M. A. Nasr and M. K. Aouf, "Bounded convex functions of complex order." Bull. Fac. Sci. Mansoura Univ., vol. 10, pp. 513-527, 1983.

[14] M. A. Nasr and M. K. Aouf, "Bounded starlike functions of complex order." Proc. Indian Acad. Sci., vol. 92, pp. 97-102, 1983, doi: 10.1007/BF02863012.

[15] M. A. Nasr and M. K. Aouf, "Starlike function of complex order." J. Natur. Sci. Math., vol. 25, pp. $1-12,1985$.

[16] H. Orhan and E. Gunes, "Fekete-Szegö inequality for certain subclass of analytic functions." General Math., vol. 14, no. 1, pp. 41-54, 2006.

[17] V. Ravichandran, Y. Polatoglu, M. Bolcal, and A. Sen, "Certain subclasses of starlike and convex functions of complex order.” Hacettepe J. Math. Stat., vol. 34, pp. 9-15, 2005.

[18] G. S. Salagean, "Subclasses of univalent functions." Lecture Notes in Math. (Springer-Verlag), vol. 1013, pp. 362-372, 1983, doi: 10.1007/BFb0066543.

[19] T. N. Shanmugam and S. Sivasubramanian, "On the Fekete-Szegö problem for some subclasses of analytic functions.” J. Inequal. Pure Appl. Math., vol. 6, no. 2, pp. 1-6, 2005.

Authors' addresses

R. M. El-Ashwah

Damietta University, Department of Mathematics, Faculty of Science, P. O. Box New Damietta 34517, Egypt

E-mail address: r_elashwah@yahoo.com

\section{A. H. Hassan}

Egypt

Zagazig University, Department of Mathematics, Faculty of Science, P. O. Box Zagazig 44519,

E-mail address: alaahassan1986@yahoo.com 